Acta Crystallographica Section E

Structure Reports

Online

ISSN 1600-5368

\section{5,5'-Diethyl-2,2'-(triazene-1,3-diyl)- di-1,3,4-thiadiazole}

\section{Hai-Su Zeng, Lu-Na Han, Si-shun Kang, Hai-lin Li and Hai-bo Wang*}

College of Science, Nanjing University of Technology, Xinmofan Road No. 5 Nanjing, Nanjing 210009, People's Republic of China

Correspondence e-mail: wanghaibo@njut.edu.cn

Received 14 October 2008; accepted 17 October 2008

Key indicators: single-crystal X-ray study; $T=294 \mathrm{~K}$; mean $\sigma(\mathrm{C}-\mathrm{C})=0.009 \AA$; $R$ factor $=0.072 ; w R$ factor $=0.178 ;$ data-to-parameter ratio $=16.3$.

In the molecule of the title compound, $\mathrm{C}_{8} \mathrm{H}_{11} \mathrm{~N}_{7} \mathrm{~S}_{2}$, the conformation about the $\mathrm{N}=\mathrm{N}$ bond is trans and the thiadiazole rings are oriented at a dihedral angle of $2.92(3)^{\circ}$. In the crystal structure, intermolecular $\mathrm{N}-\mathrm{H} \cdots \mathrm{S}$ hydrogen bonds link the molecules into chains. There are $\pi-\pi$ contacts between the thiadiazole rings [centroid-to-centroid distances $=3.699$ (3) and 3.720 (2) ̊] .

\section{Related literature}

For general background, see: Bach et al. (1996); Clark \& Hester (1991); Taniike et al. (1996). For bond-length data, see: Allen et al. (1987).

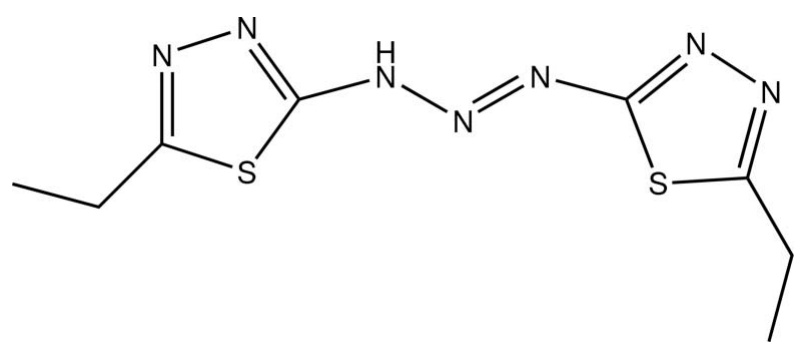

\section{Experimental}

Crystal data

$\mathrm{C}_{8} \mathrm{H}_{11} \mathrm{~N}_{7} \mathrm{~S}_{2}$

$M_{r}=269.36$

Monoclinic, $P 2_{1} / c$ $\beta=110.99(3)^{\circ}$

$V=1331.1(5) \AA^{3}$

$Z=4$

Mo $K \alpha$ radiation

Data collection

Enraf-Nonius CAD-4 diffractometer

Absorption correction: $\psi$ scan (North et al., 1968)

$T_{\min }=0.926, T_{\max }=0.962$

2431 measured reflections

Refinement

$R\left[F^{2}>2 \sigma\left(F^{2}\right)\right]=0.072$

$w R\left(F^{2}\right)=0.178$

$S=1.00$

2320 reflections

$\mu=0.39 \mathrm{~mm}^{-1}$

$T=294(2) \mathrm{K}$

$0.20 \times 0.10 \times 0.10 \mathrm{~mm}$

\author{
2320 independent reflections \\ 1468 reflections with $I>2 \sigma(I)$ \\ $R_{\text {int }}=0.0047$ \\ 3 standard reflections \\ frequency: $120 \mathrm{~min}$ \\ intensity decay: none
}

142 parameters

$\mathrm{H}$-atom parameters constrained

$\Delta \rho_{\max }=0.62{\mathrm{e} \AA^{-3}}^{-3}$

$\Delta \rho_{\min }=-1.06 \mathrm{e}^{-3}$

Table 1

Hydrogen-bond geometry $\left(\AA{ }^{\circ}\right)$.

\begin{tabular}{lllll}
\hline$D-\mathrm{H} \cdots A$ & $D-\mathrm{H}$ & $\mathrm{H} \cdots A$ & $D \cdots A$ & $D-\mathrm{H} \cdots A$ \\
\hline $\mathrm{N} 5-\mathrm{H} 5 A \cdots \mathrm{S} 2^{\mathrm{i}}$ & 0.86 & 2.84 & $3.631(4)$ & 154
\end{tabular}

Symmetry code: (i) $x,-y+\frac{1}{2}, z-\frac{1}{2}$.

Data collection: CAD-4 Software (Enraf-Nonius, 1989); cell refinement: $C A D-4$ Software; data reduction: XCAD4 (Harms \& Wocadlo, 1995); program(s) used to solve structure: SHELXS97 (Sheldrick, 2008); program(s) used to refine structure: SHELXL97 (Sheldrick, 2008); molecular graphics: ORTEP-3 for Windows (Farrugia, 1997) and PLATON (Spek, 2003); software used to prepare material for publication: SHELXTL (Sheldrick, 2008).

Supplementary data and figures for this paper are available from the IUCr electronic archives (Reference: HK2554).

\title{
References
}

Allen, F. H., Kennard, O., Watson, D. G., Brammer, L., Orpen, A. G. \& Taylor, R. (1987). J. Chem. Soc. Perkin Trans. 2, pp. S1-19.

Bach, H., Anderle, K., Fuhrmann, Th. \& Wendorff, J. H. (1996). J. Phys. Chem. 100, 4135-4140.

Clark, R. J. H. \& Hester, R. E. (1991). Advances in Materials Science Spectroscopy. New York: John Wiley \& Sons.

Enraf-Nonius (1989). CAD-4 Software. Enraf-Nonius, Delft, The Netherlands.

Farrugia, L. J. (1997). J. Appl. Cryst. 30, 565.

Harms, K. \& Wocadlo, S. (1995). XCAD4. University of Marburg, Germany.

North, A. C. T., Phillips, D. C. \& Mathews, F. S. (1968). Acta Cryst. A24, 351359.

Sheldrick, G. M. (2008). Acta Cryst. A64, 112-122.

Spek, A. L. (2003). J. Appl. Cryst. 36, 7-13.

Taniike, K., Matsumoto, T., Sato, T., Ozaki, Y., Nakashima, K. \& Iriyama, K. (1996). J. Phys. Chem. 100, 15508-15516. 


\section{supporting information}

Acta Cryst. (2008). E64, o2166 [doi:10.1107/S1600536808033941]

\section{5,5'-Diethyl-2,2'-(triazene-1,3-diyl)di-1,3,4-thiadiazole}

\section{Hai-Su Zeng, Lu-Na Han, Si-shun Kang, Hai-lin Li and Hai-bo Wang}

\section{S1. Comment}

The photophysical properties of azo compounds are of large interest in the development of nonlinear optical and optical data storage materials (Bach et al., 1996; Taniike et al., 1996; Clark \& Hester, 1991). As part of our studies in this area, we report herein the synthesis and crystal structure of the title compound.

In the title compound (Fig. 1), the bond lengths (Allen et al., 1987) and angles are within normal ranges. Rings A $(\mathrm{S} 1 / \mathrm{N} 1 / \mathrm{N} 2 / \mathrm{C} 3 / \mathrm{C} 4)$ and $\mathrm{B}(\mathrm{S} 2 / \mathrm{N} 3 / \mathrm{N} 4 / \mathrm{C} 7 / \mathrm{C} 8)$ are oriented at a dihedral angle of $2.92(3)^{\circ}$. So, they are nearly coplanar. In the crystal structure, intermolecular $\mathrm{N}-\mathrm{H} \cdots \mathrm{S}$ hydrogen bonds (Table 1) link the molecules into chains (Fig. 2), in which they may be effective in the stabilization of the structure. The $\pi-\pi$ contacts between the thiadiazole rings, $\mathrm{Cg} 2 \cdots \mathrm{Cg} 2{ }^{\mathrm{i}}$ and $\mathrm{Cg} 2 \cdots \mathrm{Cg} 1^{1 i}$ [symmetry codes: (i) -x, $1-\mathrm{y}$, -z; (ii) $-\mathrm{x},-\mathrm{y},-\mathrm{z}$, where $\mathrm{Cg} 1$ and $\mathrm{Cg} 2$ are the centroids of the rings $A(\mathrm{~S} 1 / \mathrm{N} 1 / \mathrm{N} 2 / \mathrm{C} 3 / \mathrm{C} 4)$ and $\mathrm{B}(\mathrm{S} 2 / \mathrm{N} 3 / \mathrm{N} 4 / \mathrm{C} 7 / \mathrm{C} 8)$, respectively] may further stabilize the structure, with centroidcentroid distances of 3.699 (3) and 3.720 (2) $\AA$, respectively.

\section{S2. Experimental}

For the preparation of the title compound, 5-amino-1,3,4-thiadiazole $(5 \mathrm{mmol})$ was dissolved by heating in concentrated $\mathrm{HCl}(50 \mathrm{ml})$ in a water bath, after which the solution was cooled to $268 \mathrm{~K}$ and a solution of sodium nitrite $(2.5 \mathrm{mmol})$ in water $(3.5 \mathrm{ml})$ was added dropwise with stirring. The resulting bright-yellow diazonium solution was allowed to stand in ice for $30 \mathrm{~min}$, after which a saturated solution of sodium acetate $(100 \mathrm{ml}, \mathrm{pH}=5)$ was added. The precipitate was removed by filtration, and purified by crystallization from toluene. Crystals suitable for X-ray analysis were obtained by slow evaporation of an ethanol solution.

\section{S3. Refinement}

$\mathrm{H}$ atoms were positioned geometrically, with $\mathrm{N}-\mathrm{H}=0.86 \AA$ (for $\mathrm{NH}$ ) and $\mathrm{C}-\mathrm{H}=0.97$ and $0.96 \AA$ for methylene and methyl $\mathrm{H}$, respectively, and constrained to ride on their parent atoms with $\mathrm{U}_{\mathrm{iso}}(\mathrm{H})=\mathrm{xU}_{\mathrm{eq}}(\mathrm{C}, \mathrm{N})$, where $\mathrm{x}=1.5$ for methyl $\mathrm{H}$ and $\mathrm{x}=1.2$ for all other $\mathrm{H}$ atoms. 


\section{supporting information}

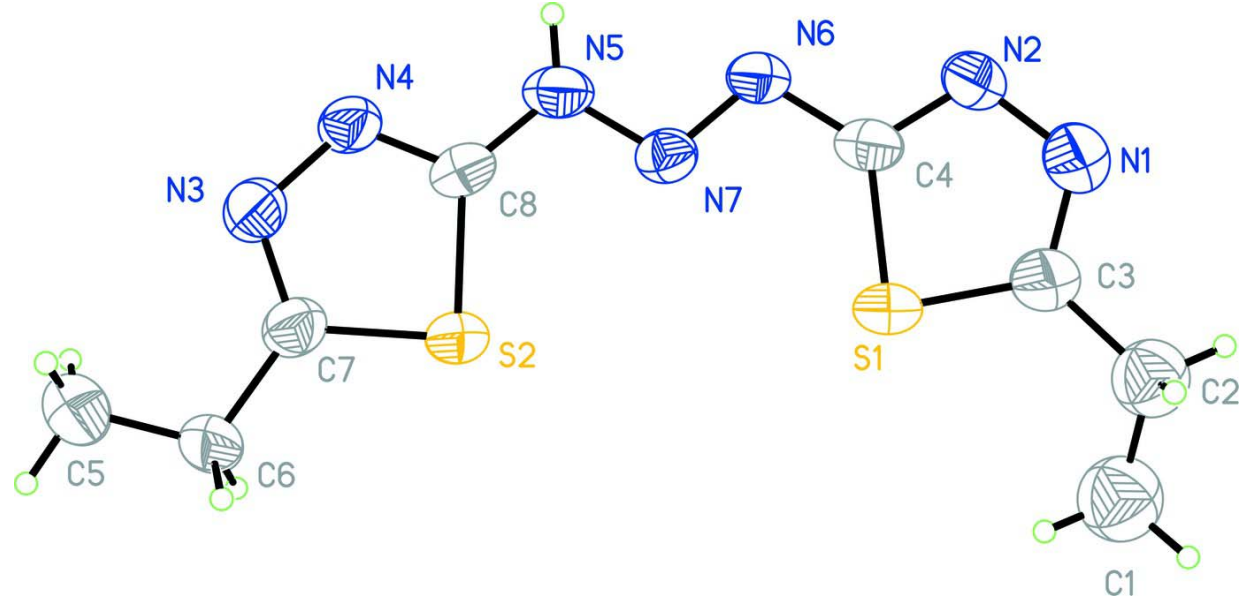

\section{Figure 1}

The molecular structure of the title molecule, with the atom-numbering scheme. Displacement ellipsoids are drawn at the $30 \%$ probability level. 


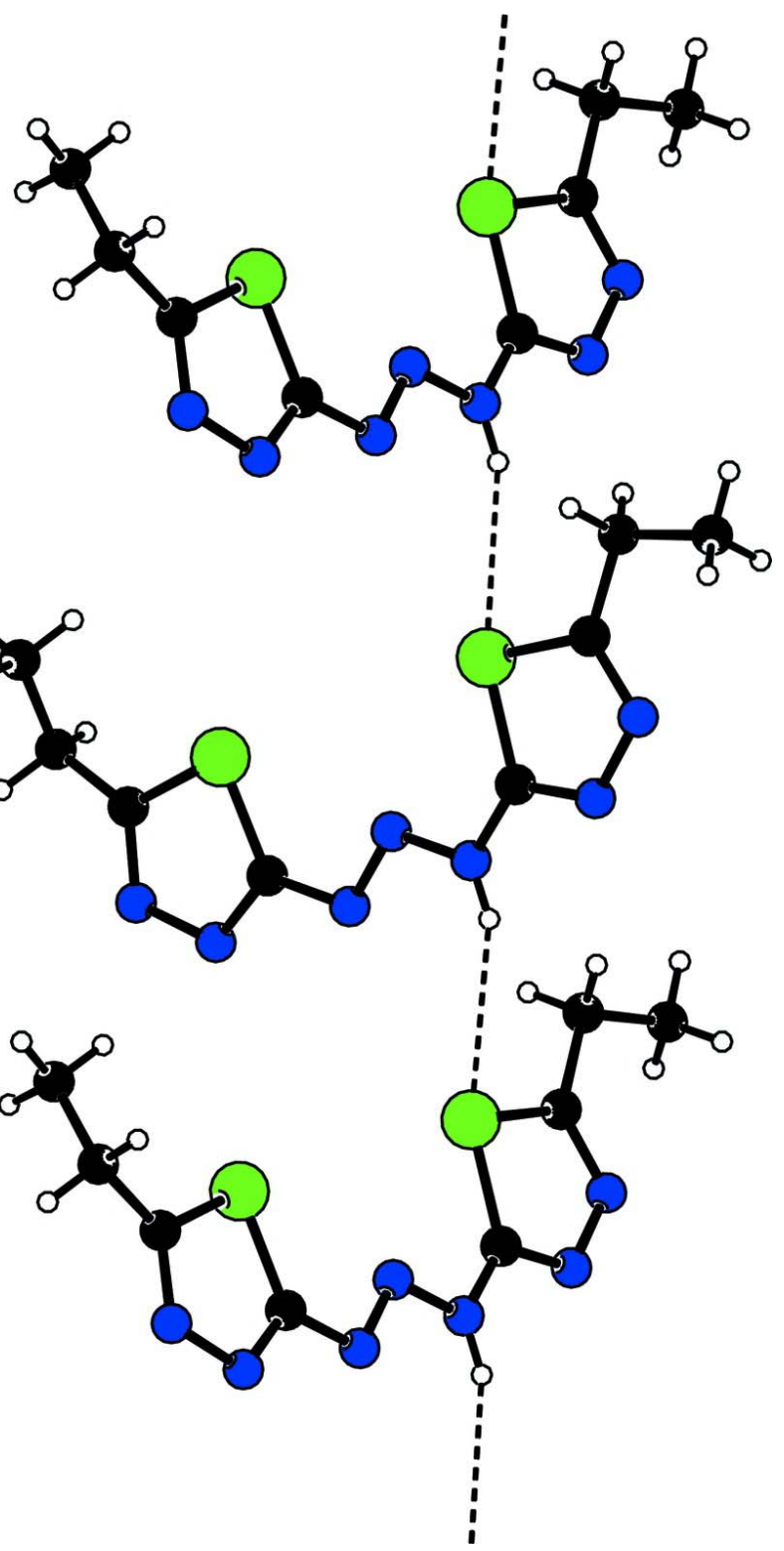

Figure 2

A partial packing diagram of the title compound.

\section{5,5'-Diethyl-2,2'-(triazene-1,3-diyl)di-1,3,4-thiadiazole}

Crystal data

$\mathrm{C}_{8} \mathrm{H}_{11} \mathrm{~N}_{7} \mathrm{~S}_{2}$

$M_{r}=269.36$

Monoclinic, $P 2_{1} / c$

Hall symbol: -P 2ybc

$a=12.188(2) \AA$

$b=9.1460(18) \AA$

$c=12.790(3) \AA$

$\beta=110.99(3)^{\circ}$

$V=1331.1(5) \AA^{3}$

$Z=4$
$F(000)=560$

$D_{\mathrm{x}}=1.344 \mathrm{Mg} \mathrm{m}^{-3}$

Mo $K \alpha$ radiation, $\lambda=0.71073 \AA$

Cell parameters from 25 reflections

$\theta=9-12^{\circ}$

$\mu=0.39 \mathrm{~mm}^{-1}$

$T=294 \mathrm{~K}$

Block, yellow

$0.20 \times 0.10 \times 0.10 \mathrm{~mm}$ 


\section{Data collection}

Enraf-Nonius CAD-4 diffractometer

Radiation source: fine-focus sealed tube Graphite monochromator $\omega / 2 \theta$ scans

Absorption correction: $\psi$ scan

(North et al., 1968)

$T_{\min }=0.926, T_{\max }=0.962$

2431 measured reflections

\section{Refinement}

Refinement on $F^{2}$

Least-squares matrix: full

$R\left[F^{2}>2 \sigma\left(F^{2}\right)\right]=0.072$

$w R\left(F^{2}\right)=0.178$

$S=1.00$

2320 reflections

142 parameters

0 restraints

Primary atom site location: structure-invariant direct methods
2320 independent reflections

1468 reflections with $I>2 \sigma(I)$

$R_{\text {int }}=0.005$

$\theta_{\max }=25.3^{\circ}, \theta_{\min }=1.8^{\circ}$

$h=-14 \rightarrow 13$

$k=0 \rightarrow 10$

$l=0 \rightarrow 14$

3 standard reflections every $120 \mathrm{~min}$ intensity decay: none

Secondary atom site location: difference Fourier map

Hydrogen site location: inferred from neighbouring sites

$\mathrm{H}$-atom parameters constrained

$w=1 /\left[\sigma^{2}\left(F_{\mathrm{o}}^{2}\right)+(0.06 P)^{2}+3.61 P\right]$

where $P=\left(F_{\mathrm{o}}^{2}+2 F_{\mathrm{c}}{ }^{2}\right) / 3$

$(\Delta / \sigma)_{\max }<0.001$

$\Delta \rho_{\max }=0.62$ e $\AA^{-3}$

$\Delta \rho_{\min }=-1.06$ e $\AA^{-3}$

\section{Special details}

Geometry. All e.s.d.'s (except the e.s.d. in the dihedral angle between two 1.s. planes) are estimated using the full covariance matrix. The cell e.s.d.'s are taken into account individually in the estimation of e.s.d.'s in distances, angles and torsion angles; correlations between e.s.d.'s in cell parameters are only used when they are defined by crystal symmetry. An approximate (isotropic) treatment of cell e.s.d.'s is used for estimating e.s.d.'s involving 1.s. planes.

Refinement. Refinement of $F^{2}$ against ALL reflections. The weighted $R$-factor $w R$ and goodness of fit $S$ are based on $F^{2}$, conventional $R$-factors $R$ are based on $F$, with $F$ set to zero for negative $F^{2}$. The threshold expression of $F^{2}>\sigma\left(F^{2}\right)$ is used only for calculating $R$-factors $(\mathrm{gt})$ etc. and is not relevant to the choice of reflections for refinement. $R$-factors based on $F^{2}$ are statistically about twice as large as those based on $F$, and $R$-factors based on ALL data will be even larger.

Fractional atomic coordinates and isotropic or equivalent isotropic displacement parameters $\left(\AA^{2}\right)$

\begin{tabular}{lllll}
\hline & $x$ & $y$ & $z$ & $U_{\text {iso }} * / U_{\text {eq }}$ \\
\hline S1 & $1.26701(14)$ & $0.49714(19)$ & $0.57391(10)$ & $0.0675(5)$ \\
S2 & $0.96258(11)$ & $0.18590(15)$ & $0.56999(10)$ & $0.0488(4)$ \\
N1 & $1.3578(5)$ & $0.6450(6)$ & $0.4508(4)$ & $0.0804(16)$ \\
N2 & $1.2634(4)$ & $0.5731(5)$ & $0.3737(3)$ & $0.0548(11)$ \\
N3 & $0.7840(4)$ & $0.0373(5)$ & $0.4403(3)$ & $0.0554(12)$ \\
N4 & $0.8301(3)$ & $0.1075(5)$ & $0.3694(3)$ & $0.0512(11)$ \\
N5 & $0.9719(4)$ & $0.2610(5)$ & $0.3544(3)$ & $0.0515(11)$ \\
H5A & 0.9482 & 0.2556 & 0.2825 & $0.062^{*}$ \\
N6 & $1.1099(3)$ & $0.4124(5)$ & $0.3558(3)$ & $0.0463(10)$ \\
N7 & $1.0651(3)$ & $0.3422(4)$ & $0.4200(3)$ & $0.0417(9)$ \\
C1 & $1.5073(6)$ & $0.6346(8)$ & $0.7641(5)$ & 0.092 \\
H1B & 1.5528 & 0.7039 & 0.8189 & $0.138^{*}$ \\
H1C & 1.5570 & 0.5558 & 0.7586 & $0.138^{*}$ \\
H1D & 1.4453 & 0.5968 & 0.7861 & $0.138^{*}$ \\
C2 & $1.4558(5)$ & $0.7082(7)$ & $0.6535(5)$ & 0.073
\end{tabular}




\begin{tabular}{lllll} 
H2A & 1.5195 & 0.7411 & 0.6308 & $0.088^{*}$ \\
H2B & 1.4135 & 0.7943 & 0.6626 & $0.088^{*}$ \\
C3 & $1.3715(6)$ & $0.6141(9)$ & $0.5586(5)$ & $0.090(2)$ \\
C4 & $1.2070(4)$ & $0.4920(6)$ & $0.4245(4)$ & $0.0466(12)$ \\
C5 & $0.7047(6)$ & $-0.0851(8)$ & $0.6080(5)$ & $0.0790(19)$ \\
H5B & 0.6879 & -0.1221 & 0.6709 & $0.119^{*}$ \\
H5C & 0.7149 & -0.1654 & 0.5640 & $0.119^{*}$ \\
H5D & 0.6406 & -0.0251 & 0.5629 & $0.119^{*}$ \\
C6 & $0.8141(5)$ & $0.0036(7)$ & $0.6486(4)$ & $0.0589(14)$ \\
H6B & 0.8787 & -0.0567 & 0.6950 & $0.071^{*}$ \\
H6C & 0.8043 & 0.0837 & 0.6941 & $0.071^{*}$ \\
C7 & $0.8436(4)$ & $0.0651(6)$ & $0.5493(4)$ & $0.0509(13)$ \\
C8 & $0.9231(4)$ & $0.1912(6)$ & $0.4186(4)$ & $0.0465(12)$ \\
\hline
\end{tabular}

Atomic displacement parameters $\left(\AA^{2}\right)$

\begin{tabular}{lllllll}
\hline & $U^{11}$ & $U^{22}$ & $U^{\beta 3}$ & $U^{12}$ & $U^{13}$ & $U^{23}$ \\
\hline S1 & $0.0757(10)$ & $0.0889(12)$ & $0.0315(7)$ & $-0.0236(9)$ & $0.0116(6)$ & $0.0029(7)$ \\
S2 & $0.0510(7)$ & $0.0616(8)$ & $0.0329(6)$ & $-0.0063(7)$ & $0.0140(5)$ & $-0.0044(6)$ \\
N1 & $0.073(3)$ & $0.099(4)$ & $0.062(3)$ & $-0.029(3)$ & $0.016(3)$ & $0.013(3)$ \\
N2 & $0.053(3)$ & $0.066(3)$ & $0.043(2)$ & $-0.006(2)$ & $0.015(2)$ & $0.011(2)$ \\
N3 & $0.045(2)$ & $0.074(3)$ & $0.049(3)$ & $-0.006(2)$ & $0.019(2)$ & $-0.012(2)$ \\
N4 & $0.044(2)$ & $0.074(3)$ & $0.037(2)$ & $-0.005(2)$ & $0.0164(19)$ & $-0.010(2)$ \\
N5 & $0.052(3)$ & $0.069(3)$ & $0.033(2)$ & $0.004(2)$ & $0.014(2)$ & $0.002(2)$ \\
N6 & $0.049(2)$ & $0.057(3)$ & $0.032(2)$ & $-0.002(2)$ & $0.0141(19)$ & $0.0018(19)$ \\
N7 & $0.038(2)$ & $0.049(2)$ & $0.036(2)$ & $0.0040(18)$ & $0.0114(18)$ & $-0.0001(18)$ \\
C1 & 0.092 & 0.092 & 0.092 & 0.000 & 0.033 & 0.000 \\
C2 & 0.073 & 0.073 & 0.073 & 0.000 & 0.026 & 0.000 \\
C3 & $0.083(4)$ & $0.126(6)$ & $0.047(3)$ & $-0.045(4)$ & $0.008(3)$ & $0.010(4)$ \\
C4 & $0.047(3)$ & $0.059(3)$ & $0.034(2)$ & $0.007(3)$ & $0.015(2)$ & $0.005(2)$ \\
C5 & $0.084(4)$ & $0.095(5)$ & $0.065(4)$ & $-0.030(4)$ & $0.035(3)$ & $-0.004(4)$ \\
C6 & $0.067(3)$ & $0.072(4)$ & $0.044(3)$ & $-0.019(3)$ & $0.029(3)$ & $-0.012(3)$ \\
C7 & $0.048(3)$ & $0.061(3)$ & $0.043(3)$ & $-0.004(3)$ & $0.015(2)$ & $-0.010(2)$ \\
C8 & $0.045(3)$ & $0.053(3)$ & $0.035(2)$ & $0.001(2)$ & $0.007(2)$ & $-0.006(2)$ \\
& & & & & & \\
\hline
\end{tabular}

Geometric parameters $\left(\stackrel{A}{ }{ }^{\circ}\right)$

\begin{tabular}{llll}
\hline $\mathrm{S} 1-\mathrm{C} 3$ & $1.728(6)$ & $\mathrm{C} 1-\mathrm{H} 1 \mathrm{~B}$ & 0.9600 \\
$\mathrm{~S} 1-\mathrm{C} 4$ & $1.786(5)$ & $\mathrm{C} 1-\mathrm{H} 1 \mathrm{C}$ & 0.9600 \\
$\mathrm{~S} 2-\mathrm{C} 7$ & $1.766(5)$ & $\mathrm{C} 1-\mathrm{H} 1 \mathrm{D}$ & 0.9600 \\
$\mathrm{~S} 2-\mathrm{C} 8$ & $1.820(5)$ & $\mathrm{C} 2-\mathrm{C} 3$ & $1.541(8)$ \\
$\mathrm{N} 1-\mathrm{N} 2$ & $1.384(6)$ & $\mathrm{C} 2-\mathrm{H} 2 \mathrm{~A}$ & 0.9700 \\
$\mathrm{~N} 1-\mathrm{C} 3$ & $1.358(7)$ & $\mathrm{C} 2-\mathrm{H} 2 \mathrm{~B}$ & 0.9700 \\
$\mathrm{~N} 2-\mathrm{C} 4$ & $1.328(6)$ & $\mathrm{C} 4-\mathrm{N} 6$ & $1.399(6)$ \\
$\mathrm{N} 3-\mathrm{N} 4$ & $1.384(6)$ & $\mathrm{C} 5-\mathrm{C} 6$ & $1.487(7)$ \\
$\mathrm{N} 3-\mathrm{C} 7$ & $1.346(6)$ & $\mathrm{C} 5-\mathrm{H} 5 \mathrm{~B}$ & 0.9600 \\
$\mathrm{~N} 4-\mathrm{C} 8$ & $1.325(6)$ & $\mathrm{C} 5-\mathrm{H} 5 \mathrm{C}$ & 0.9600 \\
$\mathrm{~N} 5-\mathrm{N} 7$ & $1.365(5)$ & $\mathrm{C} 5-\mathrm{H} 5 \mathrm{D}$ & 0.9600
\end{tabular}




\begin{tabular}{|c|c|c|c|}
\hline $\mathrm{N} 5-\mathrm{C} 8$ & $1.338(6)$ & $\mathrm{C} 6-\mathrm{C} 7$ & $1.544(7)$ \\
\hline N5-H5A & 0.8600 & $\mathrm{C} 6-\mathrm{H} 6 \mathrm{~B}$ & 0.9700 \\
\hline N6-N7 & $1.306(5)$ & $\mathrm{C} 6-\mathrm{H} 6 \mathrm{C}$ & 0.9700 \\
\hline $\mathrm{C} 1-\mathrm{C} 2$ & $1.486(7)$ & & \\
\hline $\mathrm{C} 3-\mathrm{S} 1-\mathrm{C} 4$ & $86.0(3)$ & $\mathrm{N} 1-\mathrm{C} 3-\mathrm{S} 1$ & $114.6(5)$ \\
\hline $\mathrm{C} 7-\mathrm{S} 2-\mathrm{C} 8$ & $88.1(2)$ & $\mathrm{C} 2-\mathrm{C} 3-\mathrm{S} 1$ & $124.5(5)$ \\
\hline $\mathrm{C} 3-\mathrm{N} 1-\mathrm{N} 2$ & $113.1(5)$ & $\mathrm{N} 2-\mathrm{C} 4-\mathrm{N} 6$ & $116.9(4)$ \\
\hline $\mathrm{C} 4-\mathrm{N} 2-\mathrm{N} 1$ & $111.2(4)$ & $\mathrm{N} 2-\mathrm{C} 4-\mathrm{S} 1$ & $115.1(4)$ \\
\hline $\mathrm{C} 7-\mathrm{N} 3-\mathrm{N} 4$ & $113.3(4)$ & $\mathrm{N} 6-\mathrm{C} 4-\mathrm{S} 1$ & $128.0(4)$ \\
\hline $\mathrm{C} 8-\mathrm{N} 4-\mathrm{N} 3$ & $115.8(4)$ & $\mathrm{C} 6-\mathrm{C} 5-\mathrm{H} 5 \mathrm{~B}$ & 109.5 \\
\hline $\mathrm{N} 7-\mathrm{N} 5-\mathrm{H} 5 \mathrm{~A}$ & 125.1 & $\mathrm{C} 6-\mathrm{C} 5-\mathrm{H} 5 \mathrm{C}$ & 109.5 \\
\hline $\mathrm{C} 8-\mathrm{N} 5-\mathrm{N} 7$ & $109.7(4)$ & $\mathrm{H} 5 \mathrm{~B}-\mathrm{C} 5-\mathrm{H} 5 \mathrm{C}$ & 109.5 \\
\hline $\mathrm{C} 8-\mathrm{N} 5-\mathrm{H} 5 \mathrm{~A}$ & 125.1 & $\mathrm{C} 6-\mathrm{C} 5-\mathrm{H} 5 \mathrm{D}$ & 109.5 \\
\hline $\mathrm{N} 7-\mathrm{N} 6-\mathrm{C} 4$ & $108.2(4)$ & $\mathrm{H} 5 \mathrm{~B}-\mathrm{C} 5-\mathrm{H} 5 \mathrm{D}$ & 109.5 \\
\hline N6-N7-N5 & $108.9(4)$ & $\mathrm{H} 5 \mathrm{C}-\mathrm{C} 5-\mathrm{H} 5 \mathrm{D}$ & 109.5 \\
\hline $\mathrm{C} 2-\mathrm{C} 1-\mathrm{H} 1 \mathrm{~B}$ & 109.5 & $\mathrm{C} 5-\mathrm{C} 6-\mathrm{C} 7$ & $110.8(4)$ \\
\hline $\mathrm{C} 2-\mathrm{C} 1-\mathrm{H} 1 \mathrm{C}$ & 109.5 & $\mathrm{C} 5-\mathrm{C} 6-\mathrm{H} 6 \mathrm{~B}$ & 109.5 \\
\hline $\mathrm{H} 1 \mathrm{~B}-\mathrm{C} 1-\mathrm{H} 1 \mathrm{C}$ & 109.5 & $\mathrm{C} 7-\mathrm{C} 6-\mathrm{H} 6 \mathrm{~B}$ & 109.5 \\
\hline $\mathrm{C} 2-\mathrm{C} 1-\mathrm{H} 1 \mathrm{D}$ & 109.5 & $\mathrm{C} 5-\mathrm{C} 6-\mathrm{H} 6 \mathrm{C}$ & 109.5 \\
\hline $\mathrm{H} 1 \mathrm{~B}-\mathrm{C} 1-\mathrm{H} 1 \mathrm{D}$ & 109.5 & $\mathrm{C} 7-\mathrm{C} 6-\mathrm{H} 6 \mathrm{C}$ & 109.5 \\
\hline $\mathrm{H} 1 \mathrm{C}-\mathrm{C} 1-\mathrm{H} 1 \mathrm{D}$ & 109.5 & $\mathrm{H} 6 \mathrm{~B}-\mathrm{C} 6-\mathrm{H} 6 \mathrm{C}$ & 108.1 \\
\hline $\mathrm{C} 1-\mathrm{C} 2-\mathrm{C} 3$ & $115.6(6)$ & $\mathrm{N} 3-\mathrm{C} 7-\mathrm{C} 6$ & $125.8(5)$ \\
\hline $\mathrm{C} 1-\mathrm{C} 2-\mathrm{H} 2 \mathrm{~A}$ & 108.4 & $\mathrm{~N} 3-\mathrm{C} 7-\mathrm{S} 2$ & $112.5(4)$ \\
\hline $\mathrm{C} 3-\mathrm{C} 2-\mathrm{H} 2 \mathrm{~A}$ & 108.4 & $\mathrm{C} 6-\mathrm{C} 7-\mathrm{S} 2$ & $121.7(4)$ \\
\hline $\mathrm{C} 1-\mathrm{C} 2-\mathrm{H} 2 \mathrm{~B}$ & 108.4 & $\mathrm{~N} 4-\mathrm{C} 8-\mathrm{N} 5$ & $118.5(4)$ \\
\hline $\mathrm{C} 3-\mathrm{C} 2-\mathrm{H} 2 \mathrm{~B}$ & 108.4 & $\mathrm{~N} 4-\mathrm{C} 8-\mathrm{S} 2$ & $110.2(4)$ \\
\hline $\mathrm{H} 2 \mathrm{~A}-\mathrm{C} 2-\mathrm{H} 2 \mathrm{~B}$ & 107.4 & $\mathrm{~N} 5-\mathrm{C} 8-\mathrm{S} 2$ & $131.3(4)$ \\
\hline $\mathrm{N} 1-\mathrm{C} 3-\mathrm{C} 2$ & $119.3(6)$ & & \\
\hline $\mathrm{C} 3-\mathrm{N} 1-\mathrm{N} 2-\mathrm{C} 4$ & $1.1(8)$ & $\mathrm{N} 4-\mathrm{N} 3-\mathrm{C} 7-\mathrm{C} 6$ & $179.9(5)$ \\
\hline $\mathrm{N} 2-\mathrm{N} 1-\mathrm{C} 3-\mathrm{C} 2$ & $-167.6(6)$ & $\mathrm{N} 4-\mathrm{N} 3-\mathrm{C} 7-\mathrm{S} 2$ & $0.9(6)$ \\
\hline $\mathrm{N} 2-\mathrm{N} 1-\mathrm{C} 3-\mathrm{S} 1$ & $-1.5(9)$ & $\mathrm{C} 5-\mathrm{C} 6-\mathrm{C} 7-\mathrm{N} 3$ & $-3.6(8)$ \\
\hline $\mathrm{C} 1-\mathrm{C} 2-\mathrm{C} 3-\mathrm{N} 1$ & $-153.7(7)$ & $\mathrm{C} 5-\mathrm{C} 6-\mathrm{C} 7-\mathrm{S} 2$ & $175.3(4)$ \\
\hline $\mathrm{C} 1-\mathrm{C} 2-\mathrm{C} 3-\mathrm{S} 1$ & $41.7(9)$ & $\mathrm{C} 8-\mathrm{S} 2-\mathrm{C} 7-\mathrm{N} 3$ & $-0.6(4)$ \\
\hline $\mathrm{C} 4-\mathrm{S} 1-\mathrm{C} 3-\mathrm{N} 1$ & $1.1(6)$ & $\mathrm{C} 8-\mathrm{S} 2-\mathrm{C} 7-\mathrm{C} 6$ & $-179.6(5)$ \\
\hline $\mathrm{C} 4-\mathrm{S} 1-\mathrm{C} 3-\mathrm{C} 2$ & $166.4(7)$ & $\mathrm{N} 3-\mathrm{N} 4-\mathrm{C} 8-\mathrm{N} 5$ & $179.6(4)$ \\
\hline $\mathrm{C} 7-\mathrm{N} 3-\mathrm{N} 4-\mathrm{C} 8$ & $-0.9(6)$ & $\mathrm{N} 3-\mathrm{N} 4-\mathrm{C} 8-\mathrm{S} 2$ & $0.4(5)$ \\
\hline $\mathrm{N} 1-\mathrm{N} 2-\mathrm{C} 4-\mathrm{N} 6$ & $-179.5(4)$ & $\mathrm{N} 7-\mathrm{N} 5-\mathrm{C} 8-\mathrm{N} 4$ & $179.4(4)$ \\
\hline $\mathrm{N} 1-\mathrm{N} 2-\mathrm{C} 4-\mathrm{S} 1$ & $-0.3(6)$ & $\mathrm{N} 7-\mathrm{N} 5-\mathrm{C} 8-\mathrm{S} 2$ & $-1.6(6)$ \\
\hline $\mathrm{C} 3-\mathrm{S} 1-\mathrm{C} 4-\mathrm{N} 2$ & $-0.5(5)$ & $\mathrm{C} 7-\mathrm{S} 2-\mathrm{C} 8-\mathrm{N} 4$ & $0.1(4)$ \\
\hline $\mathrm{C} 3-\mathrm{S} 1-\mathrm{C} 4-\mathrm{N} 6$ & $178.7(5)$ & $\mathrm{C} 7-\mathrm{S} 2-\mathrm{C} 8-\mathrm{N} 5$ & $-178.9(5)$ \\
\hline $\mathrm{N} 2-\mathrm{C} 4-\mathrm{N} 6-\mathrm{N} 7$ & $-178.9(4)$ & $\mathrm{C} 4-\mathrm{N} 6-\mathrm{N} 7-\mathrm{N} 5$ & $-178.5(4)$ \\
\hline $\mathrm{S} 1-\mathrm{C} 4-\mathrm{N} 6-\mathrm{N} 7$ & $1.9(6)$ & $\mathrm{C} 8-\mathrm{N} 5-\mathrm{N} 7-\mathrm{N} 6$ & $-178.4(4)$ \\
\hline
\end{tabular}


supporting information

Hydrogen-bond geometry $\left(\AA,{ }^{\circ}\right)$

\begin{tabular}{lllll}
\hline$D-\mathrm{H} \cdots A$ & $D-\mathrm{H}$ & $\mathrm{H} \cdots A$ & $D \cdots A$ & $D-\mathrm{H}^{\cdots} A A$ \\
\hline $\mathrm{N} 5-\mathrm{H} 5 A \cdots \mathrm{S} 2^{\mathrm{i}}$ & 0.86 & 2.84 & $3.631(4)$ & 154 \\
\hline
\end{tabular}

Symmetry code: (i) $x,-y+1 / 2, z-1 / 2$. 